Voix et Images

volxetimages

\title{
« En marge de la vie » ou le roman québécois 1900-1933
}

\section{Adrien Thério}

Volume 7, numéro 1, automne 1981

\section{Adrien Thério}

URI : https://id.erudit.org/iderudit/200303ar

DOI : https://doi.org/10.7202/200303ar

Aller au sommaire du numéro

Éditeur(s)

Les Presses de l'Université du Québec

ISSN

0318-9201 (imprimé)

1705-933X (numérique)

Découvrir la revue

Citer cet article

Thério, A. (1981). « En marge de la vie » ou le roman québécois 1900-1933. Voix et Images, 7(1), 45-55. https://doi.org/10.7202/200303ar d'utilisation que vous pouvez consulter en ligne.

https://apropos.erudit.org/fr/usagers/politique-dutilisation/ 


\title{
«En marge de la vie» ou le roman québécois 1900-1933
}

\author{
par Adrien Thério
}

Pendant plusieurs années, je me suis posé des questions sur la littérature québécoise du XIXe siècle tout comme je m'en suis posé sur mes contemporains. Et voici que depuis peu je m'interroge sur l'espace littéraire un peu brumeux qui va de 1900 à 1933. Car, entre 1933 et 1939, les choses changent. Cinq ou six livres qui sont presque des chefs-d'œuvre en l'espace de six ans. S'il faut donc faire une coupure dans le temps, fixons-la à 1933, date de la parution d'Un homme et son péché de C.-H. Grignon et essayons de scruter un peu la conscience de ceux qui l'ont précédé, eux qui entraient dans le XXe siècle avec un peu plus de culture que leurs prédécesseurs et qui commençaient à mieux comprendre ce qu'était que la création. Que nous ont-ils laissé?

Disons-le franchement: pas grand'chose, même si ce pas grand'chose mérite qu'on s'y arrête pour le bénéfice de notre histoire littéraire. Evidemment, je n'ai pas lu tous les romans et récits de cette période, mais j'en ai lu suffisamment pour avoir une bonne idée des questions qu'il faut leur poser.

Au départ, il faut savoir que certains de ces romanciers sont citoyens du monde; les autres (la majorité) s'occupent de rapaillages, c'est-à-dire font partie de la bourgeoisie encanaillée qui se fait gloire de défendre le bouf et la charrue. En fait, les deux catégories de créateurs que je viens de nommer s'apparentent sans le savoir car, partisans de l'ouverture au monde dans un cas et partisans de la quétainerie nationale dans l'autre, ils sont tous aliénés, dépossédés, perdus. Ne sachant trop à quoi se rattacher, certains, par l'intermédiaire de leurs personnages, se mettront à voyager, à se donner des rudiments de culture pour oublier qu'ils viennent de nulle part; les autres clameront tant qu'ils pourront à leurs rares lecteurs, même si ces derniers sont notaires, avocats, curés, hommes d'affaire, qu'il n'y a rien de plus beau que la vie d'un habitant.

Gilles Marcotte ne s'y est pas trompé en intitulant son anthologie de la littérature québécoise qui va de 1895 à 1935 :Vaisseau d'or et Croix du chemin. Qu'est-ce que c'est que le "Vaisseau d'or" si ce n'est l'aliénation dans les deux sens du mot? La folie d'abord et la perte du souvenir ensuite, je veux dire 
l'errance parce que le pays n'a rien qui puisse nous retenir. Les «Croix du chemin" ce sont, en somme, le bœuf et la charrue ou en plus grossier - ou en plus délicat comme on voudra - la grosse quétainerie québécoise. Est-ce à dire que toute la littérature de cette période souffrirait d'aliénation? II y a évidemment quelques exceptions mais elles ne sont pas nombreuses dans la création romanesque 1900-1933.

La littérature officielle, celle des anthologies et des histoires littéraires, a conservé les noms de tous ceux qui ont illustré ce que Marcotte appelle l'exotisme et le terroirisme. J'avoue que, chez ces créateurs que j'appelle citoyens du monde, je trouve très peu de véritable exotisme. Ce sont des gens qui s'exilent parce que la vie intellectuelle, au Québec, est nulle et ils doivent par conséquent, s'ils veulent respirer un peu, aller s'alimenter ailleurs: ce sont aussi des gens de bonne famille, quelquefois de grandes familles qui vont étudier en Europe et que le "patriotisme» (en tout cas ce qu'ils appellent patriotisme) va ramener ici et qui, avant de mourir d'inanition, feront tout pour ressusciter des valeurs mortes.

Je parlerai d'abord de ces citoyens du monde et plutôt que d'interroger les plus connus comme Roquebrune et Dugas, je déterrerai des œuvres de romanciers que nous avons oubliés. Ils méritaient probablement leur sort mais il reste que la lecture de leur œuvre nous apprend beaucoup de choses sur la mentalité de l'époque.

Qu'est-ce que nous trouvons dans Au large de l'écueil d'Hector Bernier, roman d'une enflure remarquable, publié en 1912 ? Le personnage principal est un garçon d'une bonne famille bourgeoise parti étudier en Europe, on ne sait trop pourquoi. II revient chez lui pour se donner corps et âme à son pays, en ouvrant tout grand les bras à la politique, avec la bénéfiction et la protection de son père. Mais il fallait qu'il possède le monde avant de posséder son pays.

J'ai glissé sur l'onde immortelle, le soir, à travers Venise endormie; $j^{\prime}$ ai vu, des hauteurs du Pincio, le couchant inonder Rome de féérie et de splendeur, et, du sommet du Vésuve, la baie de Naples et la campagne italienne dérouler leur poésie empoignante, et j'ai vu, de la Tour Eiffel, le Paris gigantesque de mes rêves, et, à la Comédie-Française (sic), où l"on jouait "CEdipe-Roi", la résurrection de la Grèce antique ${ }^{\text {. }}$

$\mathrm{Ce}$ «je» a pris soin cependant de ne pas se corrompre au contact des autres civilisations et surtout de la France contemporaine. Jules Hébert, personnification du jeune homme bien élevé, a toutes les qualités morales et intellectuelles qu'il faudrait pour être utile à son pays. Le malheur, c'est qu'on ne sait pas très bien, après trois cents pages d'exaltations patriotiques et religieuses, comment il va s'y prendre pour rendre service a sa patrie. Ce Jules Hébert, que l'auteur a voulu préserver des mauvaises influences de l'étranger, avait déjà l'esprit dérangé avant de partir pour l'Europe ou alors, c'est l'Europe qui, l'obligeant à se ressaisir, le mène finalement sur le chemin de la folie patriotique et religieuse. Il est clair que ce garçon, qui tient tellement à nous faire croire à la sincérité de son patriotisme, vit complètement en 
dehors de la vie en faisant feu de tout bois pour oublier l'immense vide qu'il sent en lui.

Changeons d'auteur. Quelle belle idylle nous trouvons dans Élégie de Wolfred Nelson, publié en 1932 ! Deux jeunes filles, extraordinairement belles, cousines et héritières de parents riches, viennent de terminer leurs études chez les bonnes sceurs et s'amènent chez le père de l'une d'elles, passer l'été ou quelques semaines, dans sa villa au Lac des Souches, "un petit coin de paradis» dans les Laurentides. Et c'est là que ces deux jeunes filles de grandes familles canadiennes-françaises (on ne sait trop comment la richesse leur est venue, si ce n'est que Suzanne est la «fille unique d'un père ayant acquis une fortune considérable...") vont rencontrer Rosaire, fils d'un habitant voisin qui, ne se sentant pas à l'aise sur la terre de son père, passe une partie de son temps à la villa du riche citadin et lui rend divers services. Il sera donc le cicerone des deux jeunes filles. Mais Rosaire n'est pas un fils d'habitant ordinaire. II joue du violon. II a déjà pris des leçons d'un curé qui avait étudié à la ville. II veut poursuivre ses études malgré l'interdiction de son père. L'amour qu'il voue à l'une des deux cousines, même si cette dernière ne le lui rend pas, lui donnera la force de partir à Montréal pour se perfectionner. Puis, ce sera l'Europe. Quand il reviendra à Montréal, à l'occasion de son premier concert nord-américain, il sera déjà célèbre dans le monde entier. C'est dire tout le chemin parcouru. Et l'auteur-narrateur de nous dire:

Il conquiert actuellement toutes les grandes villes de la vieille Europe. Je l'ai entendu à Bruxelles, à Londres, à Paris, à Rome. Partout c'est un triomphe, un véritable engouement. On se l'arrache. Tous voudraient l'entendre en même temps. C'est un second Paganini'

Voici donc un autre garçon qui part à la conquête du monde et de la gloire. Mais pourquoi? Ne serait-ce pas parce que son pays n'a rien à lui offrir, rien à lui proposer? Et qu'est-ce qui le porte à la fin à revenir chez lui ? Conquérir. cette fois, celle qui l'avait autrefois repoussé. Il s'imagine que sa renommée va lui ouvrir toutes grandes les portes de l'amour. Mais la belle appartient à un autre. Il en sera si déchiré qu'il voudra se donner la mort et s'affaissera en plein concert. $\hat{O}$ retournement des choses, l'autre cousine était là qui veillait et, après tant d'années, elle réussira à lui faire boire la potion magique qui lui fera oublier la première. L'avant-dernier paragraphe du livre nous dit: "La cloche de la petite église sonnait à toute volée, les aubépines étaient fleuries...».

Nous sommes donc en présence d'un autre personnage qui veut posséder le monde entier mais qui est manipulé, d'un bout à l'autre du récit, par un romancier qui, incapable d'accepter la vie de tous les jours et la pauvreté du Québec d'alors, se crée un monde de richesses et oblige ses personnages à vivre selon ses lubies. Pour inventer une histoire pareille (Élégie est un roman qui se lit bien), il faut être complètement dépossédé de son pays.

Ouvrons maintenant En marge de la vie, roman de Lucie Clément, publié en 1934 et dont le titre m'a suggéré mon titre d'article. Nous sommes à Ottawa 
au moment où nous faisons connaissance avec les deux personnages importants du roman, deux jeunes femmes de bonne famille, l'une anglaise et l'autre canadienne-française. Elles sont instruites, bien éduquées, elles aiment le théâtre et se permettent des randonnées sportives. Nous sommes donc en befle compagnie. Joan, l'anglaise, est orpheline mais elle est riche puisque son père lui a laissé un bel héritage. Nicole, québécoise d'origine, se trouve un peu mal à l'aise à Ottawa. "Éprise de lectures, de romanesque, de sculpture, Nicole se révèle, dans toute sa manière d'être, attachée à sa famille, à son pays comme le chêne à la terre par ses racines». Cette phrase en elle-même en dit long sur l'auteur. La comparaison de la fin est d'autant plus mauvaise que cette Nicole qui deviendra le personnage le plus important du livre, déjà transplantée à Ottawa, décidera de partir pour l'Inde quelques mois plus tard. L'attachement au pays, c'est l'auteur qui veut nous y faire croire. Elle s'en va donc en Inde car, après être tombée amoureuse d'un Québécois qui la laisse tomber pour sa jeune sœur, elle s'éprend d'un bel Anglais, pilote dans l'aviation britannique et qu'on vient de muter à Simla, en Inde. Ici comme dans le roman précédent, nous avons affaire à des gens de la haute société qui donnent des réceptions grandioses où l'on retrouve tous les grands noms dans le domaine des affaires ou des arts. Dans l'un et l'autre livre, nous rencontrons des sculpteurs, des peintres, des musiciens et tout ce monde se mêle harmonieusement avec les puissants de la politique et de l'économie. Pour un peu, on se croirait dans une société parvenue au faîte d'une grande civilisation et qui ne trouve désormais de plaisir que dans la décadence, une décadence propre, qui n'offense pas la bonne morale.

Pourquoi ces romanciers, esprits bien équilibrés - du moins peut-on l'imaginer - essaient-ils de nous jeter tant de poudre aux yeux? Ne serait-ce pas parce qu'ils n'en peuvent plus de leur petite vie miséreuse et que le seul moyen qu'ils ont de la transformer, c'est de s'en inventer une qui leur fasse oublier la vraie? La preuve, c'est que Nicole, à Simla, trouvera le moyen, pendant les absences de son aviateur, de tomber amoureuse de son cousin André, lequel lui sera finalement ravi dans un accident de voiture. Elle quittera alors son mari pour revenir à Québec vivre en errance le reste de ses jours. Avant son départ, elle a été capable de dire à son mari: « Je vivais comme en dehors de moi, au-delà de moi-même dans une attente indécise, dans une imprécision de rêve ${ }^{3}$.» N'est-ce pas avouer très candidement son aliénation? C'est cette même femme dont la langue est plutôt imprécise qui ose dire à son Anglais: "Ta langue ne se plie pas à la subtilité de la pensée française.» Comme tous les démunis qui réussissent à se convaincre, pour oublier leur petitesse, qu'ils sont savants et riches à leur façon, ainsi Nicole a réussi à se faire accroire, un certain temps, qu'elle avait le monde à ses pieds. Même avec la complicité de l'auteur, elle ne réussit pas à cacher son désarroi. Sur le chemin du retour, le narrateur nous présente enfin la vraie Nicole, "désenchantée, morte à tout espoir».

L'impression que nous laissent tous ces livres, c'est celle d'une société 
où l'on tourne en rond, où toute forme d'espoir est mort-né en dépit des efforts que font les auteurs pour nous faire croire qu'ils vivent dans une société de nantis. Ils savent pourtant que c'est faux. Mais le plus dépossédé des dépossédés essaie toujours de se persuader qu'il est important, qu'il est puissant et qu'il peut s'offrir le monde en partage. Nous sommes dans un monde d'illusions. On pourrait me rétorquer que la littérature, c'est toujours un monde d'illusions et que les romanciers dont je viens de parler ne font pas exception à la règle. Je répondrai que ces romanciers ont créé l'illusion dans un monde d'illusions et qu'en conséquence, ils n'ont jamais pu permettre à leurs personnages d'avoir la moindre emprise sur la vie. De là vient toute la différence entre leur mensonge romanesque et celui des romanciers comme Louis Hémon et Albert Laberge, deux exceptions parmi cette génération de fabulateurs.

Si nous regardions maintenant l'autre côté de la médaille? Qu'en est-il de cette bourgeoisie encanaillée qui s'est portée à la défense du bœuf et de la charrue? Ont-ils réussi, eux, à avoir un peu plus d'emprise sur la vie? Sont-ils capables de nous présenter des personnages qui savent qui ils sont, ce qu'ils font, capables de se laisser emporter par leurs passions? Pas plus que les premiers. Eux aussi sont aliénés. Le savent-ils? Je ne crois pas. Ils s'imaginent qu'ils prêchent la rédemption à leurs ouailles sans se rendre compte que leurs églises sont vides.

Je m'explique. Tous ces "rapailleurs" qui prêchaient le retour à la terre, qui redisaient sur tous les tons que rien n'était plus beau au monde que le métier d'agriculteur, de cultivateur, qui étaient-ils? C'était des gens qui avaient passé par de bonnes écoles sinòn par l'université, qui avaient donc une bonne éducation et qui auraient été très malheureux si on les avait obligés à gagner leur vie en cultivant la terre. A qui prêchaient-ils? Car si on prêche, c'est qu'on veut convertir. Leur voix a-t-elle atteint les gens qui étaient déjà exilés aux "States» ou ceux qui préparaient leur exil? Leur voix a-t-elle touché les fils de colons ou de cultivateurs qui rêvaient des lumières de la ville et les a-t-elle convaincus d'aller s'ouvrir un lot quelque part?

Comment leur voix aurait-elle pu atteindre ces paysans puisque, pour la plupart, ils ne savaient pas lire. Et même s'ils avaient pu lire, imagine-t-on que les fils d'habitants passaient leurs soirées à lire des romans? Ces "rapailleurs" ont donc convaincu des gens qui, comme eux, avaient de l'éducation: des curés, des avocats, des politiciens, des lettrés qui étaient satisfaits de leur sort. George-Étienne Cartier, pour ne prendre qu'un exemple. préférait brasser de grosses affaires et vivre confortablement à la ville. Mais il ne manquait jamais une occasion de vanter les mérites, les grandeurs de l'agriculteur. 
Les défenseurs du bceuf et de la charrue ont donc prêché dans le vide car les lecteurs qu'ils auraient dû logiquement atteindre leur ont toujours échappé. Ils ont quand même continué à prêcher, à s'extasier sur les beautés de la campagne canadienne, à célébrer les soleils couchants sur nos vertes prairies ou nos montagnes cendrées d'or parce qu'il n'y avait rien d'autre en vue.

Perdue complètement la génération de 1900 !

La ligne de l'horizon leur est complètement coupée!

On ne sait plus où on en est. On fait de grands « sparages » pour se prouver qu'on est encore en vie. L'exaltation patriotique si bien pratiquée dans l'art oratoire au XIXe siècle, envahit l'espace romanesque. Mais les personnages passent sans presque laisser de trace. Il faut le dire, les personnages de Damase Potvin (Restons chez nous, L'Appel de la terre), de Blanche LamontagneBeauregard (La Vieille maison, Récits et Légendes), de Léo-Paul Desrosiers (Sources, Les Opiniâtres, Âme et Paysages), d'Adjutor Rivard (Chez nous), du Frère Marie-Victorin (Récits laurentiens), de l'abbé Groulx (L'Appel de la race. Les Rapaillages) sont tout aussi falots sinon plus que ceux que nous avons rencontrés chez les tenants de l'ouverture au monde. Pas un, dans tous ces récits, qui soit vraiment lui-même, qui vive pleinement ses passions, ses désirs, ses amours! On cherche en vain.

C'est probablement Damase Potvin qui aura été le plus convaincu de tous ces rapailleurs. II a publié je ne sais plus combien de récits, romans et contes pour chanter la gloire du paysan québécois. La gloire du paysan québécois! Est-ce possible? Quand on y regarde de près, ce n'est pas la condition du paysan québécois qu'il a chantée, mais une nature grandiose (selon lui) peinte sur une toile riche de couleurs. II a réussi à faire vivre un peu cette nature en la coloriant à la manière des cartes de Noël dessinées par des enfants qui rêvent aux anges. Mais ce paysan, ce colon, il n'est jamais parvenu à lui donner vie.

Inutile de dire que cette nature devant laquelle tous les romanciers se sont extasiés paraît factice. Les accents qui la chantent sont si démesurés qu'on ne peut s'empêcher de les trouver, la plupart du temps, ridicules. Je pourrais citer des passages de Au large de l'écueil d'Hector Bernier (1912). de La Terre d'Ernest Choquette (1916), de La Terre vivante d'Harry Bernard (1925) ou des Récits et Légendes de Blanche Lamontagne-Beauregard. J'ai l'embarras du choix! Dire qu'il faudra attendre des gens venus d'ailleurs comme Louis Hémon et Marie Le Franc pour humaniser et grandir cette nature; qu'il faudra un Louis Hémon et un Albert Laberge pour qu'enfin des personnages cessent de tricher avec le lecteur! 
Posons-nous maintenant cette question: comment expliquer qu'autant d'écrivains, pendant toute une période, n'aient pas réussi à vraiment donner vie à leurs personnages, à les faire vibrer dans leurs œuvres? Je n'oserai pas dire que ma réponse est la seule ni la bonne mais j'avancerai que la raison principale, c'est que ces romanciers se sont tous leurrés en s'imaginant qu'ils pouvaient créer des personnages et les faire vivre en les privant dès le départ d'une part vitale de leur être, la vie sexuelle. On n'a qu'à regarder pour voir. Évidemment, je peux nommer des douzaines de personnages qui parlent le langage de l'amour. Quand on y regarde de press, on se rend compte que leur langage est faux, qu'il est pure invention. Ainsi Jules Hébert, le héros de Au large de l'écueil, qui rencontre une Française sur le bateau qui le ramène au Québec. croira avoir trouvé la perle rare. Comme beaucoup d'autres de son espèce, il cherche le grand, l'inaccessible amour:

Oh oui, j'ai souvent rêvé à celle qui viendrait... J'ai toujours respecté ce rêve... La seule manière d'en avoir le culte, c'est de respecter toutes les femmes... Sans doute. on est fou d'espérer l'irréel, mais dites-le-moi, est-ce impossible de trouver un cœur dont le vôtre est rempli comme un vase qui déborde?... ${ }^{4}$

Maintenant, écoutons Bob Stonehaven, le bel aviateur anglais, mari de Nicole dans En marge de la vie:

Si tu maimes, pourquoi profanes-tu notre amour que je considere comme sacré? (...) Je t'avais mise au-dessus des humains, au rang des dieux. T'aurais-je porté malheur en te donnant le nom d'idole?

Comment peut-on aimer quelqu'un d'amour humain, comment peut-on rechercher la jouissance sexuelle chez son partenaire quand on tient des propos pareils? En divinisant une chose aussi humaine que l'amour, on l'anéantit. Voilà pourquoi tous ces écrivains ont privé leurs personnages de vie sexuelle.

Le fait que, à cette époque, les gens se mariaient, avaient des enfants n'est pas une preuve que nos grands-parents jouissaient de leur corps. La jouissance leur était interdite. Je suis porté à croire que les romanciers ne faisaient que reproduire dans leurs livres une situation de fait. La femme devenait pour eux une sorte de lieu sacré et tabou. On pouvait s'en approcher mais défense y pénétrer. Ce n'est pas pour rien qu'entre 1850 et 1950 , tous les personnages féminins importants de nos romans sont des vierges souffrantes, des mater dolorosa qui tournent les yeux désespérément vers le ciel. Qu'on songe à Blanche d'Habreville, à Angeline de Montbrun, à Maria Chapdelaine, à Marie, fille de Menaud, à Donalda, à Angélina, l'amoureuse du Survenant, pour n'en nommer que quelques-unes.

La lecture des mauvais livres est souvent déprimante mais elle peut aussi être très éclairante. Je reviens encore à $A u$ large de l'écueil de Hector Bernier. L'auteur nous apprend ce qui suit au sujet de son héros, Jules Hébert: 
Tandis qu'ensemencée par de vrais parents Canadiens-Français (sic). pétrie (c'est moi qui souligne) définitivement par les prêtres du Séminaire de Québec, l'âme du jeune homme était profondément chrétienne ${ }^{5}$.

C'est on ne peut plus clair: l'âme du jeune homme (on pourrait dire de tous les jeunes gens) a été pétrie (je resouligne) par les prêtres du Séminaire. On ne sera pas surpris de l'entendre dire à la page suivante:

Le papillon rose n'égara jamais ses ailes entre les quatre murs du vieux collège où je fus pensionnaire, et l'été, je courais les bois du Saguenay, les lacs des Laurentides, les champs de la ferme patriarcale, ou je louvoyais dans l'Anse de Kamouraska. La grande nature était mon amoureuse... L'Université vint, et mes jeunes amies de Québec respectèrent la sérénité de mon caur... ${ }^{6}$

La papilion rose, qu'est-ce que c'est sinon le sexe ou la vie sexuelle? Et, ce papillon rose n'ose pas entrer dans les lieux sacrés où l'on forme la jeunesse. Comment cette jeunesse pourra-t-elle s'échapper de cette enceinte pour jouir de la vie? Symboliquement parlant, c'est la castration. Or comme il faut bien continuer à vivre et tâcher de trouver des raisons de traîner son existence de par le monde, on se persuade, comme Jules Hébert, que ala grande nature est une amoureuse».

Voilà pourquoi on chante la terre paternelle, la jument grise, la terre du huitième, le ber, les premières semailles. Tout cela fait partie des éléments naturels dans lesquels on se noie.

Quant à la description de la nature elle-même, elle fait l'objet d'une ridi-

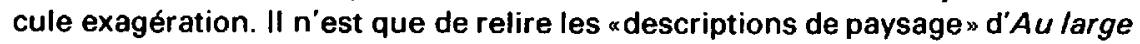
de l'écueil (au moins cent pages sur trois cents). d'Ames et Paysages de Desrosiers, de La Terre vivante de Harry Bernard et de tous les romans de Damase Potvin. Prenons un exemple chez Desrosiers:

D'abord l'avait frappé tout ce que notre pays renferme de tableaux et de scènes aux couleurs voyantes, éclatantes et crues. Les crépuscules occupaient la première place. II en avait contemplé de simples: un immense brasier dont le foyer serait caché derrière l'écran noir et ondulé des montagnes, incendiant le ciel de toutes ses lueurs, illuminant de ses langues de feu tous les nuages, flamboyant au dessus des plaines de neige blanche ou de la mer d'un bleu indigo et opaque. Quelques-uns éclaboussaient l'horizon de leurs rejaillissements, drapaient des tentures pourpres,... 7

Que dire de Marie dans La Terre vivante qui voit, en se promenant dans un champ, des «iris veloutés», des «lis jaunes», des «bermudiennes bleues», des "anémones graciles», des "gentianes», des "liserons». Pour une fille de la campagne, ce n'est pas mal! C'est dire jusqu'à quel point l'auteur tenait à nous en mettre plein la vue!

Mais cet excès n'a trompé personne. Il n'a pas empêché le lecteur de se sentir perdu dans son propre pays. Ce n'est pas la nature ou l'amour de la 
nature qui a porté tous ces romanciers et conteurs à castrer leurs personnages. C'est la société dans laquelle ils vivaient, dans laquelle ils se déplaçaient comme des automates. La société de 1900-1933, c'est l'héritière de la société du XIXe siècle. Et on sait très bien qu'à partir de 1870, après le grand combat ultramontains-libéraux, combat qui s'est terminé par l'anéantissement des libéraux, l'Église ne trouvait désormais plus d'obstacle devant elle. Elle prit sur elle de façonner comme elle l'entendait l'âme de tout un peuple. Jules Hébert employait le mot «pétrir». Ils l'ont si bien pétrie cette âme canadienne-française en lui prêchant l'horreur de tout ce qui sentait de loin ou de près la sexualité que la génération de 1900, fille dénaturée d'une génération castrée, s'est retrouvée dans une sorte d'enclos intellectuel où il n'était plus possible de faire autre chose que de tourner en rond.

Et c'est ainsi que de 1900 à 1933, des romanciers émasculés vont créer des personnages à leur image; que d'autre part d'autres inventeurs d'histoires aussi infirmes que les premiers vont s'extasier sur nos vieilles choses tant et si bien qu'ils réussiront même à chosifier leurs pauvres personnages perdus dans la grande nature.

Toute cette génération de beaux parleurs pouvait finalement se retrouver à la Relève. Les membres de cette revue ont beau être des intellectuels, ils sont fils de leurs pères. Ce sont des héritiers de la génération de 1900. La religion prend toute la place chez eux. Ils se rendent bien compte qu'ils vivent dans une société pauvre, ignorante, injuste. Que vont-ils faire pour elle? Rien. Aucune action sociale ou politique. D'abord, la révolution intérieure. Et leur idole? Maritain. La révolution intérieure, imagine-t-on?

Mais est-ce leur faute s'ils sont aussi indigents? Comment pourraient-ils s'en rendre compte puisqu'ils ont été «pétris" par un élément contre lequel ils ne pouvaient rien? Comment vouloir leur jeter la pierre? \&Le papillon rose n'égara jamais ses ailes" chez eux, comme dirait Jules Hébert. Qui aurait pu prévoir, en 1930, que de nos jours, le papillon rose allait fleureter si librement à travers nos rues et nos champs?

Dans toute cette production, quelques voix ont réussi à donner vie à leurs personnages: d'abord Louis Hémon avec Maria Chapdelaine; Albert Laberge avec $L a$ Scouine, Marie Le Franc avec Grand Louis l'innocent et finalement Rodolphe Girard avec Marie Calumet. II est facile de remarquer que nous nous trouvons en présence de romanciers qui n'étaient d'aucune façon liés à la société dans laquelle ils vivaient. Louis Hémon écrivait pour des lecteurs français. Marie Le Franc, qui eut peu de lecteurs avant de recevoir le prix Fémina, écrivait surtout pour se faire plaisir. Albert Laberge, lui, qui savait très bien qu'aucun éditeur n'accepterait de publier ses livres, les publiait lui-même pour son bon plaisir et celui de ses amis. Ces trois-là pouvaient écrire comme ils l'entendaient alors que tous les autres en étaient empêchés par la toile d'araignée solide que la société avait tissée autour d'eux. On leur avait dit que la sexualité n'avait rien à voir avec la nature humaine et ils le croyaient. 
Claude-Henri Grignon avec Un homme et son péché semble donner vie à Séraphin par le biais de l'avarice. Mais ce n'est pas tellement l'avarice qui fait que Séraphin est débordant de vie. Ce sont ses désirs sexuels. II ne peut pas les contenter, c'est vrai, mais it en est rempli. II ne peut s'empêcher de voir un beau sein, une belle fesse, un morceau de peau féminine sans se sentir tout émoustillé. Un vrai adolescent. II guette par la fente des cloisons pour avoir le plaisir de voir une jeune femme se déshabiller. Le roman ne nous le dit pas textuellement, mais on peut facilement imaginer que derrière cette cloison, il s'adonne au plaisir solitaire. On sent que les désirs sexuels, chez lui, même s'ils sont réprimés, NON PAS PAR LA MORALE MAIS PAR L'AVARICE, sont constamment présents et qu'à certains moments, ils vont presque causer sa perte. C'est ce qui le rend humain bien plus que son avarice. Dans ce sens, Un homme et son péché, c'est un livre de transition. Les désirs sexuels brûlants de Séraphin, c'est le «papilion rose» qui s'égare enfin dans les endroits les plus secrets et fait ses ravages. II faudra encore quelques années cependant pour qu'on l'accepte sans fausse gêne et sans que la conscience du romancier n'en soit troublée.

II y aurait peut-être lieu de nous attrister devant cette génération de romanciers «empêchés", mais pourquoi? Une littérature n'est pas faite seulement de chefs-d'œuvre. Elle ressemble souvent à la vie des gens d'une époque. Quand je lis par exemple, dans un récit de Blanche LamontagneBeauregard qui s'intitule Notre langue, un texte comme celui-ci:

... une pénible impression à voir ici et là, des affiches faites dans un français pitoyable. Sur un pont: "Défence de trotte", sur un petit bureau de poste: "Burau de Post», sur une maison: " $M$. un tel charretié", "M. un tel Cordonnié", "Réparateur de voitures", "Réparations de tout genres". Sur un hôtel: "Repas servis à tout heure", sur un magasin: "Réduction de toutes sorte", et que sais-je encore ${ }^{8}$ ?

de qui dois-je avoir pitié, des gens qui, n'ayant à peu près jamais été à l'école, sont encore capables d'écrire dans un français bien compréhensible même avec des fautes grammaticales ou de Blanche Lamontagne-Beauregard qui fait le procès de gens qui n'ont rien à se reprocher?

La lecture de ces romanciers de 1900-1933, ce n'est pas ce qu'il y a de plus palpitant mais cela ne veut pas dire que ce n'est pas intéressant. En fait, malgré tous ses défauts, cette période de notre littérature est dans un sens la plus attachante. C'est le cheminement de toute une civilisation qui se fait lentement à travers toutes ces figures romanesques pâles et drôlatiques quelquefois et c'est, chez tous ces créateurs, le désir de ne pas mourir pour rien. mourir pour rien. 
1. Hector Bernier, Au large de l'écueil, Québec, Imprimerie de l'Événement, 1912. p. 10-11.

2. Wolfred Nelson, Élégie, Montréal, Imprimerie Modèle Ltée, 1932, p. 107.

3. Lucie Clément, En marge de la vie. Montréal, Éditions Albert Lévesque, 1934, p. 174.

4. Au large de l"écueil, p. 139.

5. En marge de la vie, p. 74.

6. Au large de l'écueil, p. 15.

7. Léo-Paul Desrosiers, Ames et Paysages, Montréal, Editions du Devoir, 1922, p. 110.

8. Blanche Lamontagne-Beauregard, Récits et Légendes, Montréal, Librairie Beauchemin, 1924, p. 76-77. 